\title{
Pricing and Upper Price Bounds of Relax Certificates
}

Nicole Branger*
Antje Mahayni ${ }^{\ddagger}$

Judith C. Schneider ${ }^{\S}$

This version: September 5, 2008

\begin{abstract}
Relax certificates are written on multiple underlying stocks. The payoff depends on a barrier condition such that it is path-dependent. So long as none of the underlying assets crosses a lower barrier, the investor receives the payoff of a coupon bond. Otherwise, there is a cash settlement at maturity which depends on the lowest stock return. Thus, the products consist of a knock-out coupon bond and a knockin minimum option. In a Black-Scholes model setup, the price of the knock-out part can be given in closed (or semi-closed) form in the case of two underlyings, but not for more than two. Without considering the trivial case of one underlying, the price of the knock-in minimum option is to be calculated numerically. Alternatively to exact pricing, we derive semi-closed form upper price bounds. These bounds are the lowest upper price bounds which can be derived without the usage of numerical methods. In addition, the bounds are especially tight with respect to the market convention that the certificates are traded at a discount of the corresponding coupon bond. This is also illustrated with market data.
\end{abstract}

Keywords: Certificates, Exchange Option, Minimum Option, Correlation Structure, Model Risk

JEL: G13

*Finance Center Münster, Westfälische Wilhelms-Universität Münster, Universitätsstr. 14-16, D-48143 Münster, Germany, email: Nicole.Branger@wiwi.uni-muenster.de.

¥Mercator School of Management, Universität Duisburg-Essen, Lotharstr. 65, D-47057 Duisburg, email: antje.mahayni@uni-due.de.

$\S$ Mercator School of Management, Universität Duisburg-Essen, Lotharstr. 65, D-47057 Duisburg, email: judith.schneider@uni-due.de. 


\section{Introduction}

Recently, more and more structured products written on several instead of one underlying are issued. Amongst them are so-called relax certificates which can be interpreted as a generalized version of bonus certificates. While four issuers started to offer these products in 2006, more than 13 issuers are listed today. ${ }^{1}$

Normally, relax certificates ${ }^{2}$ are written on three stocks belonging to a similar market segment like blue chips or primary products. They are also traded on indices. The payoff depends on whether and when any of the underlyings reaches a lower barrier. So long as the barrier is not reached, the payments of the portfolio correspond to those of a coupon bond where the coupon payments usually range from $6 \%$ to $16 \%{ }^{3}$ However, if the lower barrier is hit, all future payments from the bond component are canceled. Instead, the investor receives a minimum option on the underlyings. Relax certificates thus combine a knock-out component (the bond) and a knock-in component (the minimum option). The time to maturity is usually smaller than thhat of ordinary bonus certificates. A typical choice are e.g. three years and three month with reference dates every 13 months or a maturity of about one year with a single reference date at maturity.

Relax certificates are advertised as follows: The bonus payments are appealing even in sideways moving and moderately bearish markets. The risk of loosing the bonus payments is low since this event is triggered by a significant loss in one of the underlying stocks. However, relax certificates are less attractive in highly bullish and highly bearish markets. In the first case, the investor would have been better off with a direct investment in the stocks. With relax certificates, she forgoes the participation in increasing stock prices. ${ }^{4}$ In

\footnotetext{
${ }^{1}$ These data has been taken from the monthly reports of the EUWAX and the monthly statistics of the DDI.

${ }^{2}$ Similar products are also called Top-10-Anleihe, Easy Relax Express, Easy Relax Bonus, MultiCapped Bonus or Aktienrelax. Furthermore, there are also relax certificates which bear some features of express certificates.

${ }^{3}$ Some examples for contracts which are traded in the market will be given in Section 5 .

${ }^{4}$ There are also certificates where the investor can participate in the development of the underlying assets if the terminal value of worst performing stock is larger than the face value of the coupon bond.
} 
extremely bearish markets, the investor is also worse off. Here, she has to participate in the (highest) losses at the stock market. This contradicts the naming 'relax'.

In this paper, we provide a detailed analysis of relax certificates. In particular, we analyze the pricing, upper price bounds, and risk management. Related literature includes Wallmeier and Diethelm (2008) and Lindauer and Seiz (2008). They analyze (multi) barrier reverse convertibles which are traded in Switzerland and are similar to the German relax certificates. Lindauer and Seiz (2008) rely on Monte Carlo simulations to price these contracts in a standard Black-Scholes framework with correlated assets. Wallmeier and Diethelm (2008) extend a multinomial tree introduced by Chen, Chung, and Yang (2002) to value barrier reverse convertibles on three underlyings. In contrast, our main focus is on closed-form or semi-closed form solutions.

Recall that relax certificates can be interpreted as a knock-out coupon bond and a knock-in minimum option. In the literature, there is an extensive analysis of barrier options. Without claiming completeness, closed-form solutions for standard barrier options are given by Rubinstein and Reiner (1991), Rich (1994) and Haug (1998). More exotic barrier options are, for example, considered in Kunitomo and Ikeda (1992) (two-sided barriers) and Heynen and Kat (1994a,b) (external barriers). For multi-asset barrier options, we refer to Wong and Kwok (2003) and Kwok, Wu, and Yu (1998). Closed-form solutions for pricing options on the minimum or maximum of two risky assets are firstly introduced in Stulz (1982). An extension to more than two risky assets is given by Johnson (1987).

The probability that at least one underlying reaches the barrier is important for pricing and risk management. In the simple case of one underlying asset, the distribution of the first hitting time is well known in a Black-Scholes setup, cf. for example Merton (1973). It can be calculated using the reflection principle, firstly introduced by Rich (1994), or the method of images. For two underlyings, a semi-closed form solution is given in He, Keirstead, and Rebholz (1998) where the distribution function is approximated by using an infinite Bessel function. Based on a more general work of Rebholz (1994), Zhou (2001) applies these results to credit risk modeling where similar problems occur. This was extended by Overbeck and Schmidt (2005) who use a deterministic time change for 
each Brownian motion. The first hitting time distribution of more than two underlyings, however, cannot be given in closed form for a general correlation structure.

Our main findings are as follows. The decomposition into a knock-out coupon bond and a knock-in minimum option is useful to understand the market convention. The contracts are designed such that relax certificates can be offered at a discount of the associated coupon bond. Formally, this gives a condition on admissible (or attractive) contract parameters in terms of the barrier and coupon payments. Basically, this condition is met if there is a lower bound on the coupon rate and/or an upper bound on the barrier level. In this case, a trivial upper price bound is given by the corresponding coupon bond. This price bound can be tightened by subtracting the price of a put option on the minimum of the underlyings with a strike price equal to the barrier.

In addition, we show that price bounds can be determined by considering subsets of the underlyings. In the extreme case where the number of underlyings is reduced to one, the upper price bound can be calculated in closed-form in a Black-Scholes setup. This price bound is decreasing in the volatility of the underlying. In particular, this implies that the lowest upper price bound is given by using the stock with the highest volatility as underlying. Due to the drawbacks of the extreme case of one-underlying which obviously contradicts the basic idea of multiple underlyings, we extensively study higher dimensions. We show that tight but still tractable price bounds result from considering all subsets consisting of two underlyings.

In order to test the practical relevance of our theoretical results, we analyze relax certificates which are currently traded at the market. For typical contract specifications, the price of relax certificates on two or three underlyings is up to $10 \%$ lower than the price of the corresponding coupon bond. The risk that at least one of the underlying stocks hits the lower barrier can thus not be neglected and is highly economically significant. We also compare the market prices to the upper price bounds which are based on two underlyings only. It turns out that the market prices are well above these upper price bounds, which confirms that these contracts are overpriced (at least relative to the model of Black-Scholes) and which also shows that the upper price bounds are rather tight. 
The remainder of the paper is organized as follows. In Section 2, the payoff structure of relax certificates is defined and analyzed. In addition, we focus on conditions on the contract parameters which are implied by the contract design. This allows us to derive model independent upper price bounds. In Section 3, we assume a Black-Scholes model and give a representation of (exact) prices as well as (model-dependent) upper price bounds. In particular, we give a tight upper price bound in semi-closed form. The dependence of the prices and price bounds on the characteristics of the underlyings is discussed in Section 4. A comparison to market prices can be found in Section 5. Section 6 concludes.

\section{Product Specification and Model Independent Price Bounds}

\subsection{Product Specification}

In general, a relax certificate is written on $n$ underlying stocks, where $n$ is equal to 2 or 3 for currently traded relax certificates. Let $S_{t}^{(j)}$ be the price of stock $j$ at time $t$. For ease of exposition, we set the initial value of all stocks equal to one, i.e. $S_{0}^{(j)}=1(j=1, \ldots, n) .{ }^{5}$

The payoff of the relax certificate depends on whether at least one of the stocks has hit its lower barrier $m(m<1)$, i.e. has lost the fraction $1-m$ of its value. Usually, $m$ is chosen to be quite low, e.g. $m=0.5$, so that this event constitutes a significant loss of this stock. The first hitting time of stock $j(j=1, \ldots, n)$ with respect to the barrier level $m$ is denoted by $\tau_{m, j}$. The first hitting time of the portfolio of all underlying stocks is denoted $\tau_{m}$, i.e.

$$
\begin{aligned}
\tau_{m, j} & :=\inf \left\{t \geq 0, S_{t}^{(j)} \leq m\right\} \\
\tau_{m}^{(n)} & :=\min \left\{\tau_{m, 1}, \ldots \tau_{m, n}\right\}
\end{aligned}
$$

If none of the underlyings reaches the level $m, \tau_{m}^{(n)}$ is set to $\tau_{m}^{(n)}=\infty$.

\footnotetext{
${ }^{5}$ This is in line with currently traded relax certificates where the minimum option is written on the return of the underlying stocks in $t_{N}$.
} 
The relax certificate can be decomposed into two parts, a knock-out (RO) and a knockin (RI) component. Its total payoff at maturity $t_{N}$ is $\mathrm{RC}_{t_{N}}^{(n)}=\mathrm{RO}_{t_{N}}^{(n)}+\mathrm{RI}_{t_{N}}^{(n)}$, where we assume that payments before maturity are accumulated at the continuously compounded risk-free rate $r .{ }^{6}$ The set of all payment dates is denoted by $\underline{\underline{T}}=\left\{t_{1}, \ldots, t_{N}\right\}^{\prime}$, the current point in time is $t_{0}<t_{i}$. If the barrier has not been hit until $t_{i} \in \underline{\underline{T}}(i=1, \ldots, N)$, the investor receives a bonus payment which can be interpreted as a coupon payment with coupon rate $\delta$. At maturity $t_{N}$, she also receives the nominal value of the certificate which we normalize to one. This part of the payoff can be interpreted as a knock-out component $\mathrm{RO}_{t_{N}}^{(n)}$

$$
\mathrm{RO}_{t_{N}}^{(n)}=\sum_{i=1}^{N} \delta e^{r\left(t_{N}-t_{i}\right)}\left(1-1_{\left\{\tau_{m}^{(n)} \leq t_{i}\right\}}\right)+\left(1-1_{\left\{\tau_{m}^{(n)} \leq t_{N}\right\}}\right)
$$

where 1 is an indicator function. If the barrier is hit before $t_{N}$, the investor forgoes all future bonus payments as well as the repayment of the nominal value. Instead, she gets an option on the minimum of the $n$ underlying stocks with maturity in $t_{N}$. The payoff from this knock-in component $\mathrm{RI}_{t_{N}}^{(n)}$ at time $t_{N}$ is given by

$$
\mathrm{RI}_{t_{N}}^{(n)}=\min \left\{S_{t_{N}}^{(1)}, \ldots, S_{t_{N}}^{(n)}\right\} 1_{\left\{\tau_{m}^{(n)} \leq t_{N}\right\}}
$$

We summarize the payoff from the relax certificate in the following definition:

Definition 1 (Relax certificate) The compounded payoff of a relax certificate with nominal value 1 , coupon rate $\delta$, lower boundary $m$, payment dates $\underline{\underline{T}}=\left\{t_{1}, \ldots, t_{N}\right\}$, and $n$ underlying stocks $S^{(1)}, \ldots, S^{(n)}$ is

$$
\begin{aligned}
R C_{t_{N}}^{(n)}= & \sum_{i=1}^{N} \delta e^{r\left(t_{N}-t_{i}\right)}\left(1-1_{\left\{\tau_{m}^{(n)} \leq t_{i}\right\}}\right)+\left(1-1_{\left\{\tau_{m}^{(n)} \leq t_{N}\right\}}\right) \\
& +\min \left\{S_{t_{N}}^{(1)}, \ldots, S_{t_{N}}^{(n)}\right\} 1_{\left\{\tau_{m}^{(n)} \leq t_{N}\right\}} .
\end{aligned}
$$

\subsection{Model Independent Price Bounds}

Relax certificates are advertised by a rather high coupon rate and a price below the price of the corresponding coupon bond. We call these relax certificates attractive:

\footnotetext{
${ }^{6}$ Throughout the paper we assume that $r$ is constant.
} 
Definition 2 (Attractive relax certificate) A relax certificate is called attractive iff

$$
\delta>r \quad \text { and } \quad R C_{t_{0}}^{(n)}<\sum_{i=1}^{N} \delta e^{-r\left(t_{i}-t_{0}\right)}+e^{-r\left(t_{N}-t_{0}\right)} .
$$

The discount as compared to the price of a coupon bond is achieved by the knock-out feature of the bond component. However, note that in case of a knock-out, the payoff is not replaced by zero but by the payoff of a minimum option. For the relax certificate to be attractive, the investor has to switch from a "higher" to a "lower" payoff in this case, i.e. the foregone future bond payments must be worth less than the minimum option. A condition to ensure that this is indeed the case is given in the following lemma:

Lemma 1 (Upper price bound: coupon bond) A sufficient condition on the contract parameters $(\delta, m)$ to ensure $R C_{t_{0}}^{(n)}<\sum_{i=1}^{N} \delta e^{-r\left(t_{i}-t_{0}\right)}+e^{-r\left(t_{N}-t_{0}\right)}$ is given by

$$
m \leq \min _{\{j=0, \ldots, n\}} \delta \sum_{i: t_{i}>t_{j}} e^{-r\left(t_{i}-t_{j}\right)}+e^{-r\left(t_{N}-t_{j}\right)}
$$

In particular, a sufficient condition for Equation (7) is given by

$$
m \leq \frac{(1+\delta) e^{-r t_{N}}}{1+e^{-r t_{N}}}
$$

PROOF: If the barrier is not hit, the payoffs are equal to that of a coupon bond. If the barrier is hit at time $\tau$, the investor forgoes the future payments from this bond and receives a minimum option instead. The value of this minimum option is bounded from above by the lowest stock price at time $\tau$, which is equal to $m .^{7}$ Condition (7) ensures that immediately after a coupon payment, the value of the coupon bond is larger than the upper price bound of the minimum option. In between the coupon dates, the price of the coupon bond increases and is thus also larger than $m$. To derive Equation (8), we start from the right hand side of the inequality:

$$
\begin{aligned}
\min _{\{j=0, \ldots, n\}} \delta \sum_{i: t_{i}>t_{j}} e^{-r\left(t_{i}-t_{j}\right)}+e^{-r\left(t_{N}-t_{j}\right)} & \geq \delta e^{-r\left(t_{N}-t_{0}\right)}+e^{-r\left(t_{N}-t_{0}\right)} \\
& \geq \frac{(1+\delta) e^{-r\left(t_{N}-t_{0}\right)}}{1+e^{-r\left(t_{N}-t_{0}\right)}}
\end{aligned}
$$

\footnotetext{
${ }^{7}$ To be more precise, in the case of gap risk due to jump or liquidity risk the lowest stock price can be lower than $m$.
} 
If $m$ is smaller than the last line, then Equation (7) holds.

Obviously, an upper price bound for an attractive relax certificate is given by the price of the corresponding coupon bond. The trivial superhedge can easily be tightened by selling some put options, which makes the price bound model dependent. ${ }^{8}$

Proposition 1 (Semi-Static Superhedge) Assume that the tupel $(\delta, m)$ satisfies Equation (8). Then, the following semi-static strategy is a superhedge for the relax certificate: At $t_{0}$, buy the corresponding coupon bond (with coupon $\delta$ and payment dates $\underline{\underline{T}}$ ) and sell a minimum put-option with underlyings $S=\left(S^{(1)}, \ldots, S^{(n)}\right)$, maturity $t_{N}$ and strike $m$. At time $\tau_{m}^{(n)}<t_{N}$, sell the coupon bond, buy back the minimum put-option, and buy the cheapest underlying asset.

Proof: Consider the case $\tau_{m}^{(n)}<t_{N}$ first. At $\tau_{m}^{(n)}$, the value of the hedge portfolio is

$$
C B_{\tau_{m}^{(n)}}-P_{\tau_{m}^{(n)}}^{M i n} \geq e^{-r\left(t_{N}-\tau_{m}^{(n)}\right)}(1+\delta)-P_{\tau_{m}^{(n)}}^{M i n},
$$

where $C B$ denotes the value of the coupon bond. The payoff of the minimum put-option at $t_{N}$ is bounded by

$$
P_{t_{N}}^{M i n}=\left[m-\min \left\{S_{t_{N}}^{(1)}, \ldots, S_{t_{N}}^{(n)}\right\}\right]^{+} \leq m
$$

so that at $\tau_{m}^{(n)}<t_{N}$ we have $P_{\tau_{m}^{(n)}}^{M i n} \leq e^{-r\left(t_{N}-\tau_{m}^{(n)}\right)} m$. With condition (8), it follows that

$$
\begin{aligned}
C B_{\tau_{m}^{(n)}}-P_{\tau_{m}^{(n)}}^{M i n} & \geq e^{-r\left(t_{N}-\tau_{m}^{(n)}\right)}(1+\delta)-e^{-r\left(t_{N}-\tau_{m}^{(n)}\right)} m \\
& =e^{-r\left(t_{N}-\tau_{m}^{(n)}\right)}(1+\delta-m) \\
& \geq m
\end{aligned}
$$

Therefore, the value of the hedge portfolio is large enough to buy the cheapest asset, which is worth $m$ at $\tau_{m}^{(n)}$. Obviously, this asset superhedges the minimum option. Finally, for $\tau_{m}^{(n)} \geq t_{N}$ we have $C B_{t_{N}}-P_{t_{N}}^{M i n}=C B_{t_{N}}$.

Corollary 1 (Upper Bound on $\mathbf{R C}_{t_{0}}^{(n)}$ ) For an attractive relax certificate, it holds that

$$
R C_{t_{0}}^{(n)} \leq \sum_{i=1}^{N} \delta e^{-r\left(t_{i}-t_{0}\right)}+e^{-r\left(t_{N}-t_{0}\right)}-P_{t_{0}}^{M i n}\left(S_{t_{N}}^{(1)}, \ldots, S_{t_{N}}^{(n)}\right) .
$$

\footnotetext{
${ }^{8}$ The semi-static superhedge is model independent excluding gap risk due to jump or liquidity risk.
} 
Proof: The proof follows immediately with Proposition 1.

The semi-static superhedge in Proposition 1 can be simplified by considering only a subset of underlyings, as will be shown in Section 3.2. Reducing the number of underlyings to one leads to a semi-static hedge where only one plain-vanilla put option instead of the more exotic minimum option is needed. The optimal choice which gives the lowest initial capital is then the most expensive put, where the high price can be due to a low stock price and/or a high volatility.

An issuer who sells the relax certificate as a substitute for selling a coupon bond might follow yet another hedging strategy. So long as the barrier is not hit, he might just refrain from hedging at all. If the barrier is hit, however, she is no longer short a coupon bond but short a minimum option. At this point in time, she might hedge her exposure to stock price risk by taking a long position in the worst performing stock, thus "paying back" the bond before its maturity date.

\section{Pricing and upper price bounds}

For the following analysis, we assume a Black-Scholes-type model setup. Each stock price $S_{t}^{(j)}$ satisfies the stochastic differential equation

$$
d S_{t}^{(j)}=\mu_{j} S_{t}^{(j)} d t+\sigma_{j} S_{t}^{(j)} d W_{t}^{(j)}
$$

where $\left\{W_{t}^{(j)}\right\}_{0 \leq t \leq T}$ is a standard Brownian motion under the real world measure $P$. The Wiener processes are in general correlated, i.e. for $i \neq j$ it holds that $\left\langle W^{(i)}, W^{(j)}\right\rangle_{t}=\rho_{i j} t$. In particular, we assume constant correlations. Note that Equation (10) implies that the dynamics of the stock prices under the risk neutral measure $Q$ are

$$
d S_{t}^{(j)}=r S_{t}^{(j)} d t+\sigma_{j} S_{t}^{(j)} d W_{t}^{Q,(j)}
$$

where $\left\{W^{Q,(j)}\right\}_{0 \leq t \leq T}$ is a standard Brownian motion under the equivalent martingale measure $Q$. The change of measure is given by

$$
\left(\frac{\partial Q^{(j)}}{\partial P}\right)_{t}=\exp \left\{-\frac{\mu^{(j)}-r}{\sigma_{j}} W_{t}^{(j)}-0.5\left(\frac{\mu^{(j)}-r}{\sigma_{j}}\right)^{2} t\right\} .
$$




\subsection{Prices of Relax Certificates}

Let $\mathrm{RC}_{t_{0}}^{(n)}$ denote the price at $t_{0}$ of the relax certificate which is written on $n$ underlying assets $S^{(1)}, \ldots, S^{(n)}$. Pricing by no arbitrage immediately gives

Proposition 2 (Price of a relax certificate) The $t_{0}$-price of a relax certificate with coupon payments $\delta$, payment dates $\underline{\underline{T}}=\left\{t_{1}, \ldots, t_{N}\right\}$ and $n$ underlying assets is given by $R C_{t_{0}}^{(n)}=R O_{t_{0}}^{(n)}+R I_{t_{0}}^{(n)}$. The prices of the components are

$$
\begin{aligned}
R O_{t_{0}}^{(n)} & =\delta \sum_{i=1}^{N} e^{-r\left(t_{i}-t_{0}\right)} Q\left(\tau_{m}^{(n)}>t_{i} \mid \mathcal{F}_{t_{0}}\right)+e^{-r\left(t_{N}-t_{0}\right)} Q\left(\tau_{m}^{(n)}>t_{N} \mid \mathcal{F}_{t_{0}}\right) \\
R I_{t_{0}}^{(n)} & =E\left[\int_{t_{0}}^{t_{N}} e^{-r\left(u-t_{0}\right)} C_{u}^{M i n, n} d N_{u} \mid \mathcal{F}_{t_{0}}\right]
\end{aligned}
$$

where $N_{t}:=1_{\left\{\tau_{m}^{(n)} \leq t\right\}}$ and $C_{t}^{M i n, n}:=E\left[e^{-r\left(t_{N}-t\right)} \min \left\{S_{t_{N}}^{(1)}, \ldots, S_{t_{N}}^{(n)}\right\} \mid \mathcal{F}_{t}\right]$.

PROOF: Pricing by no arbitrage immediately gives

$$
\begin{aligned}
\mathrm{RC}_{t_{0}}^{(n)}= & \delta \sum_{i=1}^{N} e^{-r\left(t_{i}-t_{0}\right)} Q\left(\tau_{m}^{(n)}>t_{i} \mid \mathcal{F}_{t_{0}}\right)+e^{-r\left(t_{N}-t_{0}\right)} Q\left(\tau_{m}^{(n)}>t_{N} \mid \mathcal{F}_{t_{0}}\right) \\
& +E_{Q}\left[e^{-r\left(t_{N}-t_{0}\right)} \min \left\{S_{t_{N}}^{(1)}, \ldots, S_{t_{N}}^{(n)}\right\} 1_{\left\{\tau_{m}^{(n)}<t_{N}\right\}} \mid \mathcal{F}_{t_{0}}\right] .
\end{aligned}
$$

Using iterated expectations yields

$$
\begin{aligned}
& E_{Q}\left[e^{-r\left(t_{N}-t_{0}\right)} \min \left\{S_{t_{N}}^{(1)}, \ldots, S_{t_{N}}^{(n)}\right\} 1_{\left\{\tau_{m}^{(n)}<t_{N}\right\}} \mid \mathcal{F}_{t_{0}}\right] \\
& =E_{Q}\left[\int_{t_{0}}^{t_{N}} e^{-r\left(u-t_{0}\right)} E\left[e^{-r\left(t_{N}-u\right)} \min \left\{S_{t_{N}}^{(1)}, \ldots, S_{t_{N}}^{(n)}\right\} \mid \mathcal{F}_{u}\right] 1_{\left\{\tau_{m}^{(n)} \in d u\right\}} \mid \mathcal{F}_{t_{0}}\right]
\end{aligned}
$$

With the definition of $N_{t}$, the pricing formula follows. The price of the minimum option on $n$ assets is given in Appendix $\mathrm{C}$ for $n=2$ and in Appendix $\mathrm{D}$ for general $n$.

The price of the knock-out bond component in Equation (12) depends on the distribution of the first hitting time $\tau_{m}^{(n)}$, i.e. the first time when one of the stocks hits the barrier. The price (13) of the knock-in minimum option depends on the joint distribution of the first hitting time and the stock prices at this first hitting time. In case of one underlying, the first hitting time distribution is well known and can be derived using the martingale 
stopping theorem as in Harrison (1985) or the reflection principle as in Karatzas and Shreve (1999). The price of the relax certificate can then be calculated in closed-form:

\section{Proposition 3 (Price of a relax certificate on one underlying)}

For $n=1$, the price $R C_{t_{0}}^{(n=1)}$ according to Equations (12) and (13) is given in closed form where the survival probability $Q\left(\tau_{m}^{(n=1)} \geq t\right)$ needed in Equation (12) is given by:

$$
Q\left(\tau_{m}^{(n=1)} \geq t\right)=N\left(\frac{-\ln \frac{m}{S_{0}}+\left(r-\frac{1}{2} \sigma^{2}\right) t}{\sigma \sqrt{t}}\right)+e^{2 \frac{r-\frac{1}{2} \sigma^{2}}{\sigma^{2}} \ln \frac{m}{S_{0}}} N\left(\frac{\ln \frac{m}{S_{0}}+\left(r-\frac{1}{2} \sigma^{2}\right) t}{\sigma \sqrt{t}}\right)
$$

The minimum option in Equation (13) reduces to the underlying itself, and the price of the knock-in minimum option is

$$
R I_{t_{0}}^{(1)}=m \int_{t_{0}}^{t_{N}} e^{-r\left(u-t_{0}\right)} Q\left(\tau_{m}^{(n=1)} \in d u \mid \mathcal{F}_{t_{0}}\right)
$$

where $Q\left(\tau_{m}^{(n=1)} \in d u \mid \mathcal{F}_{t_{0}}\right)$ is given in Appendix $A$.

Proof: Equation (14) is based on well known results. For the sake of completeness, these are given in Appendix A. With respect to Equation (15), first note that

$$
\begin{aligned}
C_{t}^{\mathrm{Min}, n=1} & =E_{Q}\left[e^{-r\left(t_{N}-t\right)} \min \left\{S_{t_{N}}^{(1)}\right\} \mid \mathcal{F}_{t}\right] \\
& =E_{Q}\left[e^{-r\left(t_{N}-t\right)} S_{t_{N}}^{(1)} \mid \mathcal{F}_{t}\right]=S_{t}^{(1)}
\end{aligned}
$$

In addition, we know that for $\tau_{m}^{(1)}=u$ it holds that $S_{u}=m$. This gives

$$
\begin{aligned}
E\left[\int_{t_{0}}^{t_{N}} e^{-r\left(u-t_{0}\right)} C_{u}^{\mathrm{Min}, n=1} d N_{u} \mid \mathcal{F}_{t_{0}}\right] & =m E\left[\int_{t_{0}}^{t_{N}} e^{-r\left(u-t_{0}\right)} d N_{u} \mid \mathcal{F}_{t_{0}}\right] \\
& =m \int_{t_{0}}^{t_{N}} e^{-r\left(u-t_{0}\right)} Q\left(\tau_{m}^{(n=1)} \in d u \mid \mathcal{F}_{t_{0}}\right) .
\end{aligned}
$$

For more than one underlying, closed-form solutions for Equations (12) and (13) do no longer exist in general. For the special cases of uncorrelated stock prices or perfectly positively correlated stock prices, the distribution of the first hitting time follows from the one-dimensional case. For $n=2$, Zhou (2001) derives a semi-closed form solution for the first hitting time by approximating the distribution function using an infinite 
Bessel function. The price of the knock-out bond component can then be calculated in (semi-) closed form. The price of the knock-in minimum option additionally depends on the distribution of the stock prices when the barrier is hit, and for $n \geq 2$, an analytical pricing formula no longer exists in the general case.

Thus, even in the case of a simple Black-Scholes-type model setup, the prices of relax certificates have to be determined numerically. Possible methods are binomial or trinomial lattices - see e.g. Hull and White (1993) - or finite difference schemes - see e.g. Dewynne and Wilmott (1994) - which become rather time-consuming for more than one underlying. In this case, a Monte-Carlo simulation is usually preferred. However, the barrier feature causes some problems for the simulation. To get the intuition, consider, e.g., a Monte Carlo simulation with a given refinement of the timeline and a simple Euler discretization of the stock prices. If one of the stocks breaches the barrier between two discretization dates, this event might not be detected in the simulation. To control for this problem, a large number of sampling dates is needed in addition to a large number of simulation path. But still, the bias decreases very slowly, as shown by Boyle, Broadie, and Glassermann (1997), Boyle and Lau (1994) or Broadie, Glassermann, and Kou (1997). This problem is well known in the literature and analyzed by numerous authors suggesting different correction methods, like a continuity correction as proposed by Broadie, Glassermann, and Kou (1997), or the use of a (multi-dimensional) Brownian bridge as done by Beaglehole, Dybvig, and Zhou (1997) for one underlying and by Shevchenko (2003) for several underlyings.

\subsection{Upper Price Bounds}

Given that the pricing of relax certificates is subject to numerical problems, the question is whether we can find price bounds that are both easy to calculate and tight. The next proposition shows that the price of an attractive relax certificate is decreasing in the number of underlyings. Reducing the number of underlyings thus gives an upper price bound. 
Proposition 4 (Upper price bound: relax certificates on some underlyings only) Let $S=\left(S^{(1)}, \ldots, S^{(n)}\right)$ denote a set of underlyings. In addition, let $R C_{t_{0}}(\hat{S})$ denote the price of a relax certificate with coupons $\delta$, payment dates $\underline{\underline{T}}$ and underlyings $\hat{S}$ where $\hat{S} \subseteq S$. If condition (7) on the coupon rate $\delta$ holds, then

$$
R C_{t_{0}}(S) \leq R C_{t_{0}}\left(S^{\prime}\right) \text { for all } S^{\prime} \subset S .
$$

In particular, it holds that

$$
R C_{t_{0}}(S) \leq \min _{k, l \in\{1, \ldots, n\}} R C_{t_{0}}\left(S^{(k)}, S^{(l)}\right) \leq \min _{i \in\{1, \ldots, n\}} R C_{t_{0}}\left(S^{(i)}\right)
$$

Proof: Note that $\tau_{m}(S) \leq \tau_{m}\left(S^{\prime}\right)$, i.e. the 'big' certificate is knocked out no later than the 'small' one. Depending on when and whether the two certificates are knocked out, there are three cases. First, if both certificates survive until maturity, their payments coincide. Second, if both are knocked out at the same point in time, $\tau_{m}(S)=\tau_{m}\left(S^{\prime}\right) \leq t_{N}$, it holds that

$$
R C_{\tau_{m}(S)}\left(S^{\prime}\right)=C_{\tau_{m}(S)}^{\min }\left(S^{\prime}\right) \geq C_{\tau_{m}(S)}^{\min }(S)=R C_{\tau_{m}(S)}(S)
$$

Third, if the 'big' certificate is knocked out while the small one still survives, i.e. if $\tau_{m}(S) \leq t_{N}$ and $\tau_{m}(S)<\tau_{m}\left(S^{\prime}\right)$, it holds that

$$
\begin{aligned}
R C_{\tau_{m}(S)}\left(S^{\prime}\right) & \geq \min \left\{C_{\tau_{m}(S)}^{\min }\left(S^{\prime}\right), \sum_{i: t_{i}>\tau_{m}(S)} e^{-r\left(t_{i}-\tau_{m}(S)\right)} \delta+e^{-r\left(t_{N}-\tau_{m}(S)\right)}\right\} \\
& \geq \min \left\{C_{\tau_{m}(S)}^{\min }(S), \sum_{i: t_{i}>\tau_{m}(S)} e^{-r\left(t_{i}-\tau_{m}(S)\right)} \delta+e^{-r\left(t_{N}-\tau_{m}(S)\right)}\right\} \\
& \geq C_{\tau_{m}(S)}^{\min }(S)=R C_{\tau_{m}(S)}(S)
\end{aligned}
$$

where we have used that the upper bound on the price of the minimum option is lower than the price of the coupon bond, i.e. that condition (7) holds. In all three cases, the value of the 'small' certificate is at least as high as the value of the 'big' certificate, which proves the first part of the proposition. The second part then follows as a special case.

For $n=1$, this upper bound can be calculated in closed- form. For $n=2$, there is a semi-closed form solution for the knock-out component (12), and we now give an upper bound for price of the knock-in component (13). 
Proposition 5 (Upper price bound for knock-in part) For every $n \geq 2$, an upper price bound $\overline{R I}_{t_{0}}^{(n)}$ on the price of the knock-in component is given by

$$
\overline{R I}_{t_{0}}^{(n)} \leq m \int_{t_{0}}^{t_{N}} e^{-r\left(u-t_{0}\right)} Q\left(\tau_{m}^{(n)} \in d u \mid \mathcal{F}_{t_{0}}\right)
$$

In particular, for $r \geq 0$ it holds that

$$
R I_{t_{0}}^{(n)} \leq m Q\left(\tau_{m}^{(n)} \leq t_{n} \mid \mathcal{F}_{t_{0}}\right)
$$

Proof: By the law of iterated expectiations, it holds that

$$
\begin{aligned}
\mathrm{RI}_{t_{0}}^{(n)} & =E_{Q}\left[e^{-r\left(t_{N}-t_{0}\right)} \min \left\{S_{t_{N}}^{(1)}, \ldots . S_{t_{N}}^{(n)}\right\} 1_{\left\{\tau_{m}^{(n)} \leq t_{N}\right\}} \mid \mathcal{F}_{t_{0}}\right] \\
& =E_{Q}\left[E_{Q}\left[e^{-r\left(t_{N}-t_{0}\right)} \min \left\{S_{t_{N}}^{(1)}, \ldots . S_{t_{N}}^{(n)}\right\} \mid \mathcal{F}_{\tau_{m}^{(n)}}\right] 1_{\left\{\tau_{m}^{(n)} \leq t_{N}\right\}} \mid \mathcal{F}_{t_{0}}\right] \\
& =E_{Q}\left[E_{Q}\left[\min \left\{\hat{S}_{t_{N}}^{(1)}, \ldots . \hat{S}_{t_{N}}^{(n)}\right\} \mid \mathcal{F}_{\tau_{m}^{(n)}}\right] 1_{\left\{\tau_{m}^{(n)} \leq t_{N}\right\}} \mid \mathcal{F}_{t_{0}}\right]
\end{aligned}
$$

where $\hat{S}_{t}:=e^{-r\left(t-t_{0}\right)} S_{t} . \hat{S}$ is a $Q$-martingale, so that $\min \left\{\hat{S}_{t_{N}}^{(1)}, \ldots . \hat{S}_{t_{N}}^{(n)}\right\}$ is a $Q$-supermartingale. Together with the Optimal Stopping Theorem it follows that

$$
E_{Q}\left[\min \left\{\hat{S}_{t_{N}}^{(1)}, \ldots \hat{S}_{t_{N}}^{(n)}\right\} \mid \mathcal{F}_{\tau_{m}^{(n)}}\right] \leq \min \left\{\hat{S}_{\tau_{m}^{n}}^{(1)}, \ldots \hat{S}_{\tau_{m}^{n}}^{(n)}\right\}=m e^{-r\left(\tau_{m}^{(n)}-t_{0}\right)}
$$

This implies

$$
\mathrm{RI}_{t_{0}}^{(n)} \leq m \int_{t_{0}}^{t_{N}} e^{-r\left(u-t_{0}\right)} Q\left(\tau_{m}^{(n)} \in d u \mid \mathcal{F}_{t_{0}}\right)
$$

As a consequence we can state the following Theorem.

Theorem 1 (Semi closed-form upper price bound for $n \geq 2$ ) Let $n \geq 2$ and $S=$ $\left(S^{(1)}, \ldots, S^{n)}\right.$ denote the set of underlying assets. An upper price bound $\overline{R C}_{t_{0}}^{(n)}$ is given by

$$
\begin{aligned}
\overline{R C}_{t_{0}}^{(n)}=\min _{k, l \in\{1, . ., n\}}\{ & m Q\left(\min \left\{\tau_{m, k}, \tau_{m, l}\right\} \leq t_{N} \mid \mathcal{F}_{t_{0}}\right) \\
& +\delta \sum_{i=1}^{N} e^{-r\left(t_{i}-t_{0}\right)} Q\left(\min \left\{\tau_{m, k}, \tau_{m, l}\right\}>t_{i} \mid \mathcal{F}_{t_{0}}\right) \\
& \left.+e^{-r\left(t_{N}-t_{0}\right)} Q\left(\min \left\{\tau_{m, k}, \tau_{m, l}\right\}>t_{N} \mid \mathcal{F}_{t_{0}}\right)\right\}
\end{aligned}
$$


where

$$
\begin{array}{rlrl}
Q\left(\min \left\{\tau_{m, k}, \tau_{m, l}\right\} \leq t-t_{0} \mid \mathcal{F}_{t_{0}}\right) & \\
=\frac{2}{\alpha_{t}} e^{a_{1} x_{1}+a_{2} x_{2}+b\left(t-t_{0}\right)} \sum_{n=1}^{\infty} \sin \frac{n \pi \theta_{0}}{\alpha} \cdot e^{\frac{r_{0}^{2}}{2 t}} \int_{0}^{\alpha} \sin \frac{n \pi \theta}{\alpha} g_{n}(\theta) d \theta \\
a_{1}=\frac{-\alpha_{1} \sigma_{l}+\rho_{k, l} \alpha_{2} \sigma_{k}}{\left(1-\rho_{k, l}^{2}\right) \sigma_{k}^{2} \sigma_{l}} & a_{2}=\frac{-\alpha_{2} \sigma_{k}+\rho_{k, l} \alpha_{1} \sigma_{l}}{\left(1-\rho_{k, l}^{2}\right) \sigma_{l}^{2} \sigma_{k}} \\
d_{1}=a_{1} \sigma_{k}+a_{2} \sigma_{l} \rho_{k, l} & d_{2}=a_{2} \sigma_{l} \sqrt{1-\rho_{k, l}^{2}} \\
x_{1}=\log \left[\frac{S_{t_{0}}^{(1)}}{m}\right] & x_{2}=\log \left[\frac{S_{t_{0}}^{(2)}}{m}\right] \\
\alpha_{1} & =-\left(r-0.5 \sigma_{k}^{2}\right) & \alpha_{2} & =-\left(r-0.5 \sigma_{l}^{2}\right)
\end{array}
$$

and where

$$
\begin{aligned}
b & =\alpha_{1} a_{1}+\alpha a_{2}+\frac{1}{2} \sigma_{k}^{2} a_{1}^{2}+0.5 \sigma_{l}^{2} a_{2}^{2}+\rho_{k, l} \sigma_{k} \sigma_{l} a_{1} a_{2} \\
g_{n}(\theta) & =\int_{0}^{\infty} r e^{-\frac{r^{2}}{2 t}} e^{d_{1} r \sin (\theta-\alpha)-d_{2} r \cos (\theta-\alpha)} I_{\frac{n \pi}{\alpha}}\left(\frac{r r_{0}}{t-t_{0}}\right) d r .
\end{aligned}
$$

For a detailed specification of $\alpha, \theta$, and $r_{0}$, we refer to Proposition 7 in Appendix $B$.

Proof: According to Proposition 4, it holds that

$$
\mathrm{RC}_{t_{0}}^{(n)} \leq \min _{k, l \in\{1, . ., n\}}\left\{\mathrm{RC}_{t_{0}}\left(S^{(k)}, S^{(l)}\right)\right\}=\min _{k, l \in\{1, . ., n\}}\left\{\mathrm{RO}_{t_{0}}\left(S^{(k)}, S^{(l)}\right)+\mathrm{RI}_{t_{0}}\left(S^{(k)}, S^{(l)}\right)\right\}
$$

The value of the knock-out component follows from Proposition 2, while Proposition 5 gives an upper bound on the value of the knock-in minimum option. Putting the results together gives Equation (19). The probability $Q\left(\min \left\{\tau_{m, k}, \tau_{m, l}\right\} \leq t_{N}\right)$ follows from the results of He, Keirstead, and Rebholz (1998) and Zhou (2001), details are given in Appendix B.

The upper price bounds in this theorem result from subsets of two underlyings. If this upper bound is applied to a relax certificate with two underlyings, the knock-out component is priced exactly, while only the knock-in part is approximated from above. Since the "main part" of the product is explained by the knock-out part, the price bound 
will be rather tight in this case. For three or more underlyings, however, the upper price bounds will be worse, and the question is whether we can derive tighter price bounds. One possibility is to derive upper and lower bounds for the first-hitting time probabilities which can be calculated in (semi-) closed form, and then plug these bounds into the pricing equation (12) for the knock-out coupon bond and into the upper bound in Proposition 5 for the value of the knock-in minimum option. Recall that it is not possible to determine the hitting time probabilities for $n \geq 3$ in (semi-)closed form. Therefore the tightest bounds for $n=3$ which are not based on numerical approximations are achieved by using:

Lemma 2 (Upper and lower bounds for $n=3$ )

$$
\underline{Q}\left(\tau_{m}^{(n=3)} \leq t\right) \leq Q\left(\tau_{m}^{(n=3)} \leq t\right) \leq \bar{Q}\left(\tau_{m}^{(n=3)} \leq t\right)
$$

where

$$
\begin{aligned}
& \bar{Q}\left(\tau_{m}^{(n=3)} \leq t\right)=\min \{ Q\left(\min \left\{\tau_{m, 1}, \tau_{m, 2}\right\} \leq t\right)+Q\left(\tau_{m, 3} \leq t\right), \\
& Q\left(\min \left\{\tau_{m, 1}, \tau_{m, 3}\right\} \leq t\right)+Q\left(\tau_{m, 2} \leq t\right), \\
&\left.Q\left(\min \left\{\tau_{m, 2}, \tau_{m, 3}\right\} \leq t\right)+Q\left(\tau_{m, 1} \leq t\right)\right\} \\
& \underline{Q}\left(\tau_{m}^{(n=3)} \leq t\right)=\max \{ Q\left(\min \left\{\tau_{m, 1}, \tau_{m, 2}\right\} \leq t\right), \\
&\left.Q\left(\min \left\{\tau_{m, 1}, \tau_{m, 3}\right\} \leq t\right), Q\left(\min \left\{\tau_{m, 2}, \tau_{m, 3}\right\} \leq t\right)\right\} .
\end{aligned}
$$

ProOF: It holds that

$$
Q\left(\tau_{m}^{(n=3)} \leq t\right)=Q\left(\min \left\{\tau_{m, 1}, \tau_{m, 2}, \tau_{m, 3}\right\} \leq t\right)
$$

Notice that

$$
\left\{\omega \mid \min \left\{\tau_{m, 1}, \tau_{m, 2}, \tau_{m, 3}\right\} \leq t\right\}=\left\{\omega \mid \min \left\{\tau_{m, 1}, \tau_{m, 2}\right\} \leq t\right\} \cup\left\{\omega \mid \tau_{m, 3} \leq t\right\}
$$

Using

$$
P(A \cup B)=P(A)+P(B)-P(A \cap B) \leq P(A)+P(B)
$$

immediately gives the Lemma.

To derive an upper price bound on a relax certificate, $Q\left(\tau_{m}>t\right)$ is replaced by $(1-\underline{Q})$ 
while $Q\left(\tau_{m} \leq t\right)$ is replaced by $\bar{Q}$. It is straightforward to show that the resulting upper price bound is higher than the one in Theorem 1.

\section{Sensitivities}

We now take a closer look at the prices of relax certificates. In particular, we are interested in relax certificates on one and two underlyings. Their prices are upper price bounds for relax certificates on a larger number of underlyings. The first question in this context is which two underlyings to pick to get the lowest upper price bounds. The second question is how tight these price bounds are.

We provide a numerical example with respect to the model setup of Section 3. The time to maturity is 3 years, and there are two intermediate payment dates, $t_{1}=1$ and $t_{2}=2$. The bonus level is $\delta=0.11$, and the barrier is set to $m=0.5$. The relax certificate is written on two stocks with identical volatilities and a return correlation of $\rho$. The prices are calculated using a Monte-Carlo simulation with 10.000 simulation runs. To minimize the risk that we miss a hit of the barrier which happens in between to successive discretization dates, we use 100 time steps per day.

In line with market practice, we restrict the analysis to attractive relax certificates, cf. Definition 2. For these contracts, the corresponding coupon bond is more valuable than the minimum option when the barrier is hit. Intuitively, the value of the relax certificate is then the lower the higher the probability that the barrier will be hit. Its price will thus decrease both in the volatility of the underlyings and in the barrier level, while it will increase in the correlation of the two assets.

This intuition is confirmed by our numerical analysis. The upper left graph in Figure 1 shows the price of a relax certificate on two underlyings as a function of volatility and for different values of the correlation between the two assets. Furthermore, the graph shows that the impact of correlation is of the same order as the impact of the volatilities, so that a significant decrease in correlation can have a similar impact on the price as an increase in the volatility. If the correlation of the assets differs from one, the upper price bound 
based on two underlyings will thus be significantly lower than the upper price bound based on one underlying only (where the latter is equal to the price for a correlation of one).

The upper right graph shows the survival probability, i.e. the probability that the barrier will not be hit until maturity. In line with intuition, this probability is decreasing in the volatilities of the underlyings and increasing in their correlation. The lower two graphs show the value of the knock-out coupon bond and the knock-in minimum option. While the price of the knock-out bond is rather similar to the survival probability, the price of the knock-in minimum option behaves the other way round. The higher the volatility and the lower the correlation, the higher the contribution of the minimum option to the overall price of the relax certificate, and the less similar the certificate is to a standard coupon bond.

\section{Market Comparison}

\subsection{Contract Specifications}

We now analyze relax certificates currently traded at the market and compare their issue prices to our price bounds. Table 1 gives the contract specifications of six typical certificates. The underlyings and their implied volatilities can be found in Table 2. All barriers are set at a rather low value $(50 \%$ or $60 \%)$, so that at least one of the underlying stocks has to loose a rather high fraction of its initial value for the coupon bond to be replaced by the minimum option. Furthermore, the bonus rate is rather high, and all certificates are attractive.

The relax certificate Z1, issued by Commerzbank, is written on two stocks, namely Siemens and Daimler. The time to maturity is 14 month, and there are no intermediate payment dates. If both stocks do never fall below $50 \%$ of their initial value, the payoff from the bonus certificate is 111 Euros. In principle, the high bonus payment (as compared to the current risk-free rate) should just compensate the investor for the risk that the lower 


\begin{tabular}{|c|c|c|c|c|c|c|c|}
\hline Z & $\begin{array}{c}\text { Issue Price } \\
\text { incl. load }\end{array}$ & $n$ & $t_{N}$ & $\delta$ & $m$ & $\begin{array}{c}\text { Bonus payments } \\
t_{i}\end{array}$ & $\left\{r_{i}\right\}_{i=1, . .}$ \\
\hline Z1 & 101.00 & 2 & 14 months & 0.11 & $50 \%$ & at maturity & 0.0505 \\
Z2 & 101.00 & 3 & 17 month 4 days & 0.16 & $50 \%$ & at maturity & 0.0505 \\
Z3 & 101.00 & 3 & 3 years 3 months & 0.10 & $50 \%$ & every 13 months & $0.0505,0.0484,0.0472$ \\
Z4 & 101.00 & 3 & 15 month 2 days & 0.19 & $60 \%$ & at maturity & 0.0505 \\
Z4 & 101.00 & 3 & 3 years & 0.30 & $50 \%$ & at maturity & 0.0472 \\
Z6 & 1000.00 & 3 & 20 months & 0.20 & $60 \%$ & at maturity & 0.0484 \\
\hline
\end{tabular}

Table 1: Summary of traded product specifications and interest rates

barrier is hit, in which case he receives the worst of the two stocks at maturity.

The relax certificate Z2, issued by HSBC Trinkaus, is additionally written on EON. It has both a longer time to maturity and a higher bonus payment, but the same initial price as Z1. Theoretically, the higher bonus payment is thus set in such a way as to exactly offset the lower value resulting from the longer time to maturity. The third relax certificate, Z3, is issued by HVB. It is written on three stocks, namely Allianz, BASF and Deutsche Post. Different from before, there are now to intermediate payment dates after 13 and 26 months.

In contrast to the certificates presented so far, Z4 - Z6 contain an additional component. The contracts also include a knock-out minimum call option on the underlyings with a strike price equal to the terminal payoff from the bond component. The investor thus participates in the stock market if all stocks perform well. Both Z4 and Z5 are issued by Société General. Z4 is written on three banks, namely Deutsche Bank, Commerzbank and Postbank, whereas Z5 is written on Allianz, Deutsche Telekom and DaimlerChrysler. Z6 is launched by WestLB with underlyings Allianz, Bayer and RWE. 


\begin{tabular}{||l|c|c||c|c|c||}
\hline Z & Underlyings & $\begin{array}{c}\text { Implied } \\
\text { Volatilitiy }\end{array}$ & Z & Underlyings & $\begin{array}{c}\text { Implied } \\
\text { Volatility }\end{array}$ \\
\hline Z1 & $\begin{array}{c}\text { Daimler AG } \\
\text { Siemens AG }\end{array}$ & 0.33 & Z4 & Deutsche Bank & 0.34 \\
& & & & Pommerzbank & 0.42 \\
Z2 & Daimler AG & 0.33 & Z5 & Allianz & 0.46 \\
& Siemens AG & 0.35 & & Deutsche Telekom & 0.25 \\
& EON & 0.27 & & Daimler AG & 0.33 \\
\hline Z3 & Allianz & 0.32 & Z6 & Allianz & 0.32 \\
& BASF & 0.25 & & Bayer & 0.32 \\
& Deutsche Post & 0.30 & & RWE & 0.24 \\
\hline
\end{tabular}

Table 2: Underlyings of the certificates and implied volatilities of the underlyings

\subsection{Survival Probabilities and Price Bounds}

Proposition 1 states that the coupon bond is a trivial upper price bound for an attractive relax certificate. The interest rates are inferred from the corresponding zero coupons (swaps) via bootstrapping and are given in the last column of Table 1. The resulting prices of the coupon bonds are given in Table 3. For all certificates, the issue price is significantly lower than the price of the corresponding coupon bond. The risk that at least one stock looses more than $50 \%$ respectively $40 \%$ of the initial value should thus not be neglected, and it reduces the price by $4 \%$ to $11 \%$.

To assess the risk inherent in the relax certificate, we calculate the probability that the barrier will be hit by one or two underlyings, where we set $\rho_{k, l}=0.3$ and $\sigma_{k}=\sigma_{l}=0.3 .^{9}$ The results show that adding a further underlying significantly increases the risk that the bond will be knocked out. They also confirm that the risk of a knock-out is rather high, even if we only calculate a lower bound for this knock-out probability in case $n=3$.

In the next step, we use Theorem 1 to determine the upper price bounds. Table 3

\footnotetext{
${ }^{9}$ For all certificates, the implied volatilities of at least two underlyings as given in Table 2 are above $30 \%$, so that a volatility of $\sigma=0.3$ yields a lower boundary.
} 


\begin{tabular}{||c|c|c|c|c|c|c|c|c||}
\hline Z & $n$ & Issue Price & corresp. & \multicolumn{2}{|c|}{ Survival probability } & \multicolumn{3}{|c}{ Upper price bound } \\
& & incl. load & $\begin{array}{c}\text { coupon } \\
\text { bond }\end{array}$ & one & two & Knock-out & Knock-in & Price \\
\hline Z1 & 2 & 101.00 & 104,65 & $96,88 \%$ & $93,60 \%$ & 97,32 & 3,20 & 100,52 \\
Z2 & 3 & 101.00 & 107,99 & $94,97 \%$ & $89,90 \%$ & 96,11 & 5,25 & 101,36 \\
Z3 & 3 & 101.00 & 112,83 & $80,76 \%$ & $65,99 \%$ & 75,19 & 17,05 & 92,24 \\
Z4 & 3 & 101.00 & 111,72 & $87,58 \%$ & $77,19 \%$ & 86,24 & 13,69 & 99,93 \\
Z5 & 3 & 101.00 & 112,83 & $82,47 \%$ & $68,67 \%$ & 77,48 & 15,69 & 93,17 \\
Z6 & 3 & 1000.00 & 1107,37 & $81,18 \%$ & $67,85 \%$ & 751,10 & 192,90 & 944,00 \\
\hline
\end{tabular}

Table 3: Relax certificates traded at the market

The table gives the price of the corresponding coupon bond, the survival probabilities based on one and two underlyings, and the upper price bounds based on two underlyings. The calculations are based on a volatility of $\sigma=0.3$ and a correlation of $\rho=0.3$.

gives the upper price bounds, again based on $\rho_{k, l}=0.3$ and $\sigma_{k}=\sigma_{l}=0.3$. Note that for all certificates, the price of the knock-out coupon bond by far exceeds the upper bound on the value of the knock-in minimum option. Furthermore, the resulting upper price bound is below the issue price for all but one certificates. If we included dividend payments on the stocks - which we have ignored up to now - the upper price bound would even decrease further. For Z1 and Z2, we also recalculated the upper price bounds using the implied volatilities of the underlyings and varying the correlation from -1 to 1 . The price bounds are shown in Figure 2. For Z2, the issue price now exceeds the upper price bound for all correlation levels. For Z1, the issue price is below the upper bound only if we assume a correlation larger than 0.85 .

There are two possible conclusions. First, we may conclude that relax certificates are overpriced in the market. This is in line with the empirical results of Wallmeier and Diethelm (2008) for the swiss certificate market. Furthermore, the mispricing is the higher the higher the bonus payments (and thus also the higher the discount due to the knock-out feature of the bond). We conjecture that the investors do not correctly estimate the risk 
associated with the barrier feature, but overweight the sure coupon. Second, the model of Black-Scholes may not be the appropriate choice, but we may have to use a model with stochastic volatility, jumps, and maybe also a stochastic correlation between the assets instead. This is left to future research.

\section{Conclusion}

Relax certificates can be decomposed into a knock-out coupon bond and a knock-in minimum option on all underlying stocks. The contracts are designed such that relax certificates can be offered at a discount compared to the associated coupon bond. Formally, this gives a condition on admissible (or attractive) contract parameters in terms of the barrier and coupon payments.

The knock-out/knock-in event takes place when the worst-performing of the $n$ underlying stocks hits a lower barrier, which is usually quite low. Nevertheless, our analysis shows that the probability of a knock-out can not be neglected and induces a significant price discount of the relax certificate as compared to the corresponding coupon bond. The risk is the larger the higher the volatility of the underlyings, the lower their correlation, and the larger the number of stocks the certificate is written on.

In general, numerical methods are needed to price relax certificates, and even in the Black-Scholes model, closed form solutions exist for one underlying only. However, closedform or semi-closed form solutions are available for upper price bounds. A trivial upper price bound is given by the corresponding coupon bond. Furthermore, the price of a relax certificate on several underlyings is bounded from above by the price of the (cheapest) relax certificate on a subset of underlyings. We show that two underlyings are convenient to achieve meaningful and tractable price bounds. The most likely candidates to give this lowest upper price bound are the relax certificates on the most risky assets and/or the assets with the lowest correlation between the underlyings.

Finally, we test the practical relevance of our theoretical results by comparing the price bounds with market data. The upper price bounds are calculated based on the 
implied volatilities of call options on the respective underlyings. It turns out that the relax certificates which are currently traded at the market are significantly overpriced. This result is true for all correlation scenarios. 


\section{A First Hitting Time - One-Dimensional Case}

To derive the distribution of the first hitting time in the one-dimensional case, we use some results given in He, Keirstead, and Rebholz (1998). They consider the probability density and distribution function of the maximum or minimum of a one-dimensional Brownian motion with drift.

Along the lines of He, Keirstead, and Rebholz (1998), we define

$$
\underline{X}_{t}:=\min _{0 \leq S \leq t} X_{s} \quad \bar{X}_{t}:=\max _{0 \leq S \leq t} X_{s}
$$

where $X_{t}=\alpha t+\sigma W_{t}, t \geq 0$ and $\alpha, \sigma$ are constants, and $W$ is a Brownian motion defined on some probability space.

Proposition 6 Define the functions $G(x, t ; \alpha)$ and $g\left(y, x, t ; \alpha_{1}\right)$ by

$$
\begin{aligned}
G(x, t ; \alpha) & =N\left(\frac{x-\alpha t}{\sigma \sqrt{t}}\right)-e^{\frac{2 \alpha x}{\sigma^{2}}} N\left(\frac{-x-\alpha t}{\sigma \sqrt{t}}\right) \\
g\left(y, x, t ; \alpha_{1}\right) & =\frac{1}{\sigma \sqrt{t}} \phi\left(\frac{x-\alpha_{1} t}{\sigma \sqrt{t}}\right)\left(1-e^{-\frac{4 x^{2}-4 x 4 y}{2 \sigma^{2} t}}\right)
\end{aligned}
$$

where $N$ denotes the cumulative distribution function of the standard normal distribution and $\phi(z)$ the density of the standard normal distribution.

For $x \geq 0$, it holds that

$$
P\left(\bar{X}_{t} \leq x\right)=G(x, t ; \alpha) \quad P\left(X_{1}(t) \in d y, \bar{X}_{1}(t) \leq x\right)=g\left(y, x, t ; \alpha_{1}\right) d y .
$$

For $x<0$, it holds that

$$
P\left(\underline{X}_{t} \geq x\right)=G(-x, t ;-\alpha) \quad P\left(X_{1}(t) \in d y, \underline{X}_{1}(t) \geq x\right)=g\left(-y,-x, t ;-\alpha_{1}\right) d y .
$$

PROOF: c.f. He, Keirstead, and Rebholz (1998), Theorem 1, and its proof.

Corollary 2 For constant $\mu$ and $\sigma$, set

$$
S_{t}=S_{0} e^{\left(\mu-\frac{1}{2} \sigma^{2}\right) t+\sigma W_{t}}
$$


where $W$ is a Brownian motion defined on some probability space. For the first hitting time of $m<S_{0}, \tau_{m}:=\inf \left\{t \geq 0 \mid S_{t} \leq m\right\}$, it holds that

$$
\begin{aligned}
P\left(\tau_{m} \leq t\right) & =N\left(\frac{\ln \frac{m}{S_{0}}-\left(\mu-\frac{1}{2} \sigma^{2}\right) t}{\sigma \sqrt{t}}\right)+e^{2 \frac{\mu-\frac{1}{2} \sigma^{2}}{\sigma^{2}} \ln \frac{m}{S_{0}}} N\left(\frac{\ln \frac{m}{S_{0}}+\left(\mu-\frac{1}{2} \sigma^{2}\right) t}{\sigma \sqrt{t}}\right) \\
P\left(\tau_{m}^{(n=1)} \in d t\right) & =\frac{-\ln \frac{m}{S_{0}}}{\sqrt{2 \pi \sigma^{2} t^{3}}} e^{-\frac{1}{2} \frac{\left(\ln \frac{m}{S_{0}}-\left(\mu-\frac{1}{2} \sigma^{2}\right) t\right.}{\sigma^{2} t}} d t .
\end{aligned}
$$

Proof: It holds that

$$
\tau_{m}:=\inf \left\{t \geq 0 \mid S_{t} \leq m\right\}=\inf \left\{t \geq 0 \mid \ln \frac{S_{t}}{S_{0}} \leq \ln \frac{m}{S_{0}}\right\}
$$

Let $X_{t}$ denote the logarithm of the normalized asset price, i.e.

$$
X_{t}:=\ln \frac{S_{t}}{S_{0}}=\left(\mu-\frac{1}{2} \sigma^{2}\right) t+\sigma W_{t}
$$

and set $\alpha=\mu-\frac{1}{2} \sigma^{2}$. The stopping time $\tau_{m}$ is related to the first hitting time of a one-dimensional Brownian motion with drift $\alpha$. With $x:=\ln \frac{m}{S_{0}}<0$ it holds that

$$
P\left(\tau_{m} \leq t\right)=P\left(\underline{X}_{t} \leq x\right)=1-P\left(\underline{X}_{t} \geq x\right) \text {. }
$$

According to Proposition 7, we have

$$
\begin{aligned}
1-P\left(\underline{X}_{t} \geq x\right) & =1-G(-x, t ;-\alpha) \\
& =1-N\left(\frac{-x+\alpha t}{\sigma \sqrt{t}}\right)+e^{\frac{2 \alpha x}{\sigma^{2}}} N\left(\frac{x+\alpha t}{\sigma \sqrt{t}}\right) \\
& =N\left(\frac{x-\alpha t}{\sigma \sqrt{t}}\right)+e^{\frac{2 \alpha x}{\sigma^{2}}} N\left(\frac{x+\alpha t}{\sigma \sqrt{t}}\right) .
\end{aligned}
$$

Plugging in $\alpha$ and $x$ gives the distribution function. To derive the density function, define

$$
f(t):=N\left(\frac{x-\alpha t}{\sigma \sqrt{t}}\right)+e^{\frac{2 \alpha x}{\sigma^{2}}} N\left(\frac{x+\alpha t}{\sigma \sqrt{t}}\right) .
$$

Taking partial derivatives gives

$$
\begin{aligned}
f^{\prime}(t)= & N^{\prime}\left(\frac{x-\alpha t}{\sigma \sqrt{t}}\right) \times\left(\frac{-\alpha \sigma \sqrt{t}-\frac{\sigma}{2 \sqrt{t}}(x-\alpha t)}{\sigma^{2} t}\right) \\
& +e^{\frac{2 \alpha x}{\sigma^{2}}} \times N^{\prime}\left(\frac{x+\alpha t}{\sigma \sqrt{t}}\right) \times\left(\frac{\alpha \sigma \sqrt{t}-\frac{\sigma}{2 \sqrt{t}}(x+\alpha t)}{\sigma^{2} t}\right)
\end{aligned}
$$


Using $N^{\prime}(x)=\frac{1}{\sqrt{2 \pi}} e^{-\frac{1}{2} x^{2}}$, we get

$$
\begin{aligned}
e^{\frac{2 \alpha x}{\sigma^{2}}} N^{\prime}\left(\frac{x+\alpha t}{\sigma \sqrt{t}}\right) & =\frac{1}{\sqrt{2 \pi}} e^{\frac{2 \alpha x}{\sigma^{2}}-\frac{1}{2}\left(\frac{x+\alpha t}{\sigma \sqrt{t}}\right)^{2}} \\
& =\frac{1}{\sqrt{2 \pi}} e^{\frac{1}{\sigma^{2}}\left[2 \alpha x-\frac{1}{2 t}(x+\alpha t)^{2}\right]} \\
& =\frac{1}{\sqrt{2 \pi}} e^{-\frac{1}{2} \frac{(x-\alpha t)^{2}}{\sigma^{2} t}} \\
& =N^{\prime}\left(\frac{x-\alpha t}{\sigma \sqrt{t}}\right)
\end{aligned}
$$

Plugging this into the equation for $f^{\prime}(t)$ gives

$$
\begin{aligned}
f^{\prime}(t) & =N^{\prime}\left(\frac{x-\alpha t}{\sigma \sqrt{t}}\right) \times \frac{-\sigma x}{\sqrt{t} \sigma^{2} t}(x-\alpha t+x+\alpha t) \\
& =\frac{-x}{\sqrt{2 \pi \sigma^{2} t^{3}}} e^{-\frac{1}{2} \frac{(x-\alpha t)^{2}}{\sigma^{2} t}} .
\end{aligned}
$$

Using $\alpha=\mu-\frac{1}{2} \sigma^{2}$ and $x=\ln \frac{m}{S_{0}}$, which implies $\frac{\partial x}{\partial m}=\frac{S_{0}}{m}$, gives the result.

\section{B First Hitting Time - Two Dimensional Case}

The distribution of the first hitting time in the two-dimensional case is based on He, Keirstead, and Rebholz (1998) and Zhou (2001):

Proposition 7 Let $X_{t}^{(j)}=\alpha_{j} t+\sigma_{j} W_{t}^{(j)}, t \geq 0, j=1,2$, where $\alpha_{j}=-\left(\mu_{j}-\frac{1}{2} \sigma_{j}^{2}, \mu_{j}\right.$ and $\sigma_{j}$ are constants, and $W^{(1)}, W^{(2)}$ are two correlated Brownian motions with $\left\langle W^{(1)}, W^{(2)}\right\rangle_{t}=$ $\rho t$. Then, the probability that $X^{(1)}$ and $X^{(j)}$ will not hit the upper boundaries $x^{(1)}>0$ and $x^{(2)}>0$ up to time $t$ is

$$
P\left(\bar{X}_{t}^{(1)} \leq x^{(1)}, \bar{X}_{t}^{(2)} \leq x^{(2)}\right)=\frac{2}{\alpha_{t}} e^{a_{1} x_{1}+a_{2} x_{2}+b t} \sum_{n=1}^{\infty} \sin \frac{n \pi \theta_{0}}{\alpha} \cdot e^{\frac{r_{0}^{2}}{2 t}} \int_{0}^{\alpha} \sin \frac{n \pi \theta}{\alpha} g_{n}(\theta) d \theta
$$

where

$$
\left.f(r, \theta, t)=\frac{2}{\sigma_{1} \sigma_{2} \sqrt{1-\rho^{2}} \alpha t} e^{a_{1} x_{1}+a_{2} x_{2}+b t} e^{-\frac{r^{2}+r_{0}^{2}}{2 t}} \sum_{n=1}^{\infty} \sin \frac{n \pi \theta}{\alpha} \sin \frac{n \pi \theta_{0}}{\alpha} \operatorname{In\pi } \frac{r_{0} r}{t}\right) .
$$


The parameters are defined by

$$
\begin{array}{ll}
a_{1}=\frac{-\alpha_{1} \sigma_{2}+\rho \alpha_{2} \sigma_{1}}{\left(1-\rho^{2}\right) \sigma_{1}^{2} \sigma_{2}} & a_{2}=\frac{-\alpha_{2} \sigma_{1}+\rho \alpha_{1} \sigma_{2}}{\left(1-\rho^{2}\right) \sigma_{2}^{2} \sigma_{1}} \\
d_{1}=a_{1} \sigma_{1}+a_{2} \sigma_{2} \rho & d_{2}=a_{2} \sigma_{2} \sqrt{1-\rho^{2}}
\end{array}
$$

and by

$$
\begin{aligned}
& b=\alpha_{1} a_{1}+\alpha a_{2}+\frac{1}{2} \sigma_{1}^{2} a_{1}^{2}+0.5 \sigma_{2}^{2} a_{2}^{2}+\rho \sigma_{1} \sigma_{2} a_{1} a_{2} \\
& \alpha=\left\{\begin{array}{cc}
\tan ^{-1}\left(-\frac{\sqrt{1-\rho^{2}}}{\rho}\right) & \text { if } \rho<0 \\
\pi+\tan ^{-1}\left(-\frac{\sqrt{1-\rho^{2}}}{\rho}\right) & \text { otherwise }
\end{array}\right. \\
& \theta_{0}=\left\{\begin{array}{cl}
\tan ^{-1}\left(\frac{\frac{x 2}{\sigma_{2}} \sqrt{1-\rho^{2}}}{\frac{x 1}{\sigma_{1}}-\rho \frac{x 2}{\sigma_{2}}}\right) & \text { if }(.)>0 \\
\pi+\tan ^{-1}\left(\frac{\frac{x 2}{\sigma_{2}} \sqrt{1-\rho^{2}}}{\frac{x 1}{\sigma_{1}}-\rho \frac{x 2}{\sigma_{2}}}\right) & \text { otherwise }
\end{array}\right. \\
& r_{0}=x_{2} / \sigma_{2} \sin \left(\theta_{0}\right) .
\end{aligned}
$$

The function $g_{n}$ is defined as

$$
g_{n}(\theta)=\int_{0}^{\infty} r e^{-\frac{r^{2}}{2 t}} e^{d_{1} r \sin (\theta-\alpha)-d_{2} r \cos (\theta-\alpha)} I_{\frac{n \pi}{\alpha}}\left(\frac{r r_{0}}{t}\right) d r .
$$

Proof: Recall that

$$
S_{t}^{(j)}=S_{0} e^{\left(r-\frac{1}{2} \sigma_{j}^{2}\right) t+\sigma_{j} W_{t}^{(j)}}
$$

and use that

$$
\begin{aligned}
\tau_{m}^{(j)} & :=\inf \left\{t \geq 0 \mid S_{t}^{(j)} \leq m_{j}\right\} \\
& =\inf \left\{t \geq 0 \mid \ln \frac{S_{t}^{(j)}}{S_{0}^{j)}} \leq \ln \frac{m_{j}}{S_{0}^{(j)}}\right\} \\
& =\inf \left\{t \geq 0 \mid-\ln \frac{S_{t}^{(j)}}{S_{0}^{(j)}} \geq \ln \frac{S_{0}^{(j)}}{m_{j}}\right\}
\end{aligned}
$$

For $m_{j}<S_{0}^{(j)}$, it holds that $\ln \frac{S_{0}^{(j)}}{m_{j}}>0$. Let $X_{t}^{(j)}$ denote the logarithm of the normalized asset prices, i.e.

$$
X_{t}^{(j)}=-\ln \frac{S_{t}^{(j)}}{S_{0}^{(j)}}=-\left(\left(\mu_{j}-\frac{1}{2} \sigma_{j}^{2}\right) t+\sigma_{j} W_{t}\right)
$$


The stopping time $\tau_{m}$ is equal to the first hitting time of the positive upper barriers $x_{1}$ and $x_{2}$ by the two-dimensional Brownian motion $\left(X^{(1)}, X^{(2)}\right)$ with drift parameters

$$
\alpha_{1}=-\left(\mu-\frac{1}{2} \sigma_{1}^{2}\right), \quad \alpha_{2}=-\left(\mu-\frac{1}{2} \sigma_{2}^{2}\right)
$$

where the upper barriers are

$$
x_{1}=\ln \frac{S_{0}^{(1)}}{m}, \quad x_{2}=\ln \frac{S_{0}^{(2)}}{m}
$$

For the rest of the proof, see Zhou (2001).

\section{Pricing Formula of a Minimum Option based on two underlyings}

The price of the minimum option on two underlying assets with a strike price equal to zero is given in Stulz (1982):

$$
\begin{aligned}
C_{t}^{\operatorname{Min}}(n=2) & =E_{Q}\left[e^{-r\left(t_{N}-t\right)} \min \left\{S_{t_{N}}^{(1)}, S_{t_{N}}^{(2)}\right\} \mid \mathcal{F}_{t}\right] \\
& =S_{t}^{(1)}-S_{t}^{(1)} N\left(d_{1}\right)+S_{t}^{(2)} N\left(d_{2}\right)
\end{aligned}
$$

where

$$
d_{1}=\frac{\ln \frac{S_{t}^{(1)}}{S_{t}^{(2)}}+\frac{1}{2} \tilde{\sigma}^{2}\left(t_{N}-t\right)}{\tilde{\sigma} \sqrt{t_{N}-t}}, \quad d_{2}=d_{1}-\tilde{\sigma} \sqrt{t_{N}-t}, \quad \tilde{\sigma}=\sqrt{\sigma_{1}^{2}+\sigma_{2}^{2}-2 \rho \sigma_{1} \sigma_{2}} .
$$

PROOF: The risk-neutral pricing equation is

$$
E_{Q}\left[e^{-r\left(t_{N}-t\right)}\left(S_{t_{N}}^{(1)}-\max \left\{S_{t_{N}}^{(1)}-S_{t_{N}}^{(2)}, 0\right\}\right) \mid \mathcal{F}_{t}\right]
$$

The rest of the proof immediately follows from Magrabe (1978), who gives an explicit formula for the option to exchange one asset for another. 


\section{Pricing Formula of a Minimum Option based on several underlyings}

To price a minimum option on several underlyings, we rely on the results of Johnson (1987). Johnson (1987) argues that the price of a minimum option on several assets can be derived using the ansatz of Magrabe (1987) for pricing exchange options. Taking each asset as a numeraire, the price of a minimum call option on several assets with strike $K$ equals:

$$
\begin{aligned}
& C_{\text {min }}=S_{1} N_{n}\left(d_{1}\left(S_{1}, K, \sigma_{1}^{2}\right),-d_{1}^{\prime}\left(S_{1}, S_{2}, \sigma_{12}^{2}\right), \ldots,\right. \\
& \left.-d_{1}^{\prime}\left(S_{1}, S_{N}, \sigma_{1 N}^{2}\right),-\rho_{112},-\rho_{113}, \ldots,-\rho_{123}, \ldots\right) \\
& +S_{2} N_{n}\left(d_{1}\left(S_{2}, K, \sigma_{1}^{2}\right),-d_{1}^{\prime}\left(S_{2}, S_{1}, \sigma_{12}^{2}\right), \ldots,\right. \\
& \left.-d_{1}^{\prime}\left(S_{2}, S_{N}, \sigma_{2 N}^{2}\right),-\rho_{212},-\rho_{223}, \ldots,-\rho_{213}, \ldots\right) \\
& +\ldots \\
& +S_{N} N_{n}\left(d_{1}\left(S_{N}, K, \sigma_{N}^{2}\right),-d_{1}^{\prime}\left(S_{N}, S_{1}, \sigma_{N 2}^{2}\right), \ldots,\right. \\
& \left.-d_{1}^{\prime}\left(S_{N}, S_{N-1}, \sigma_{N N-1}^{2}\right),-\rho_{N 1 N},-\rho_{N 2 N}, \ldots,-\rho_{N 12}, \ldots\right) \\
& -K e^{-r T} N_{n}\left(d_{2}\left(S_{1}, K, \sigma_{1}^{2}\right), d_{2}\left(S_{2}, K, \sigma_{2}^{2}\right), \ldots,\right. \\
& \left.d_{2}\left(S_{N}, K, \sigma_{N}^{2}\right), \rho_{12}, \rho_{13}, \ldots\right) .
\end{aligned}
$$

The functions $d_{1}, d_{1}^{\prime}, d_{2}$, and $d_{2}^{\prime}$ are defined by

$d_{1}^{\prime}\left(S_{i}, S_{j}, \sigma_{i j}^{2}\right)=\frac{\log \frac{S_{i}}{S_{j}}+\frac{1}{2} \sigma_{i j}^{2} T}{\sigma_{i j} \sqrt{T}}, \quad d_{2}\left(S_{i}, K, \sigma_{i}^{2}\right)=\frac{\log \frac{S_{i}}{K}+\left(r-\frac{1}{2} \sigma_{i}^{2}\right) T}{\sigma_{i} \sqrt{T}}, \quad d_{1}=d_{2}+\sigma_{i} \sqrt{T}$.

The correlation coefficient $\rho_{i i}$ follows from

$$
\underbrace{\operatorname{Cov}\left(\log S_{i}^{*}, \log \frac{S_{i}^{*}}{S_{j}^{*}}\right)}_{\sigma_{i} \sigma_{i j} \rho_{i i j}}=\operatorname{Var}\left(\log S_{i}^{*}\right)-\operatorname{Cov}\left(\log S_{i}^{*}, \log S_{j}^{*}\right)=\sigma_{i}^{2}-\rho_{i j} \sigma_{i} \sigma_{j}
$$

which gives $\rho_{i i j}=\frac{\sigma_{i}-\rho_{i j} \sigma_{j}}{\sigma_{i j}}$. The correlation coefficient $\rho_{i j k}$ follows from

$$
\operatorname{Cov}\left(\log \frac{S_{i}^{*}}{S_{k}^{*}}, \log \frac{S_{i}^{*}}{S_{j}^{*}}\right)=\sigma_{i}^{2}-\rho_{i j} \sigma_{i} \sigma_{j}-\rho_{i k} \sigma_{i} \sigma_{k}+\rho_{j k} \sigma_{j} \sigma_{k}
$$

which gives $\rho_{i j k}=\frac{\sigma_{i}^{2}-\rho_{i j} \sigma_{i} \sigma_{j}-\rho_{i k} \sigma_{i} \sigma_{k}+\rho_{j k} \sigma_{k} \sigma_{j}}{\sigma_{i j} \sigma_{i k}}$. 


\section{References}

Beaglehole, D., P. Dybvig, and G. Zhou, 1997, Going to extremes: Correcting Simulation Bias in Exotic Option Valuation, Financial Analysts Journal January/February, 62-68.

Boyle, P., M. Broadie, and P. Glassermann, 1997, Monte Carlo methods for security pricing, Journal of Economic Dynamics and Control 21, 1267-1321.

Boyle, P., and S. Lau, 1994, Bumping up against the barrier with the binomial method, Journal of Derivatives 1, 6-14.

Broadie, M., P. Glassermann, and S. Kou, 1997, A Continuity Correction for Discrete Barrier Options, Mathematical Finance 7, 325-348.

Chen, R., S. Chung, and T. Yang, 2002, Option pricing in a multi-asset, complete market economy, Journal of Financial and Qunatitative Analysis 37, 649-666.

Dewynne, J., and P. Wilmott, 1994, Partial to Exotics, Risk December, 53-57.

Harrison, J., 1985, Browian Motion and Stochastic Flow Systems. (Wiley).

Haug, E.G., 1998, The Complete Guide to Option Pricing Formulas. (McGraw-Hill).

He, H., W. P. Keirstead, and J Rebholz, 1998, Double Lookbacks, Mathematical Finance $8,201-228$.

Heynen, R., and H. Kat, 1994a, Crossing Barriers, Risk 7, 46-51.

Heynen, R., and H. Kat, 1994b, Partial Barrier Options, Journal of Financial Engineering $3,253-274$.

Hull, J., and A. White, 1993, Efficient Procedures for Valuing European and American Path-Dependent Options, Journal of Derivatives Fall, 21-31.

Johnson, H., 1987, Options on the Maximum or Minimum of Several Asset, The Journal of Financial and Quantitative Analysis 22, 277-283. 
Karatzas, I., and S.E. Shreve, 1999, Brownian Motion and Stochastic Calculus. (Springer).

Kunitomo, N., and M. Ikeda, 1992, Pricing Options with curved boundaries, Mathematical Finance 2,4, 275-298.

Kwok, Y., L. Wu, and H. Yu, 1998, Pricing multi-asset options with and external barrier, International Journal of Theoretical and Applied Finance 1, 523-541.

Lindauer, T., and R. Seiz, 2008, Pricing (Multi-) Barrier Reverse Convertibles, Working Paper.

Magrabe, W., 1978, The value of an Option to exchange one asset for another, Journal of Finance 33, 177-186.

Merton, R. C., 1973, Theory of Rational Option Pricing, Bell Journal of Economics and Management Science 4, 141-183.

Overbeck, L., and W. Schmidt, 2005, Modelling default dependences with threshold models, Journal of Derivatives 12, 10-19.

Rebholz, J., 1994, Planar diffusions with applications to Mathematical Finance. (University of California).

Rich, D.R., 1994, The Mathematical Foundations of Barrier-Option Pricing Theory, Advances in Futures and Options Research 7, 267-311.

Rubinstein, M., and E. Reiner, 1991, Breaking Down the Barriers, Risk 4, 28-35.

Shevchenko, P., 2003, Adressing the bias in Monte Carlo pricing of multi-asset options with multiple barriers through discrete sampling, Journal of Computational Finance 6, $1-20$.

Stulz, R., 1982, Options on the Minimum or the Maximum of two risky assets, Journal of Financial Economics 10, 161-185.

Wallmeier, M., and M. Diethelm, 2008, Market pricing of Exotic Structured Products: The Case of Multi-Asset Barrier Reverse Convertibles in Switzerland, Working Paper. 
Wong, H., and Y. Kwok, 2003, Multi-asset barrier options and occupation time derivatives, Applied Mathematical Finance 10, 245-266.

Zhou, C, 2001, An Analysis of Default Correlations and Multiple Defaults, The Review of Financial Studies 14,2, 555-576. 

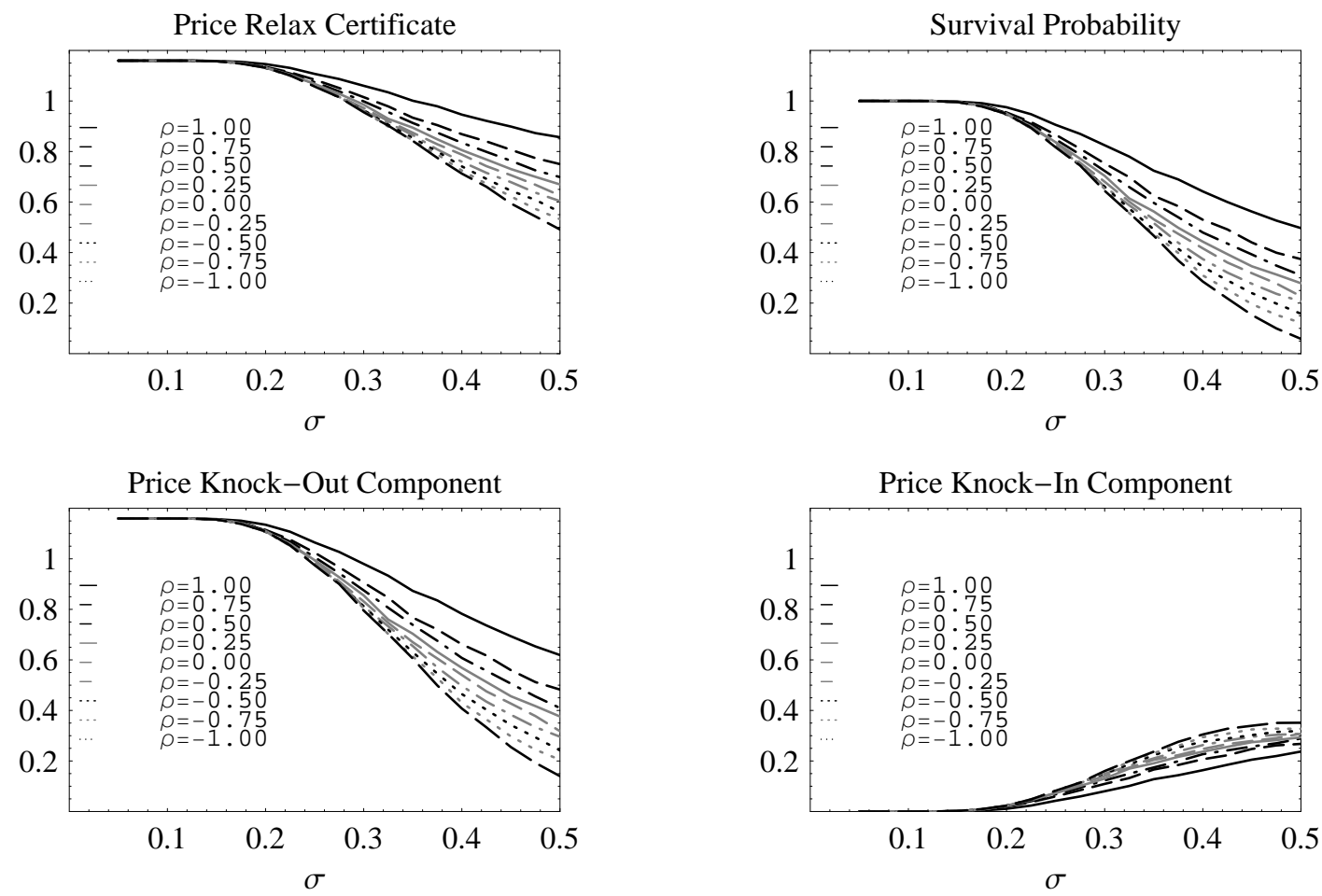

Figure 1: Relax Certificate on two underlyings

The figure analyzes a relax certificate on two underlyings as a function of the volatility $\sigma$ for varying levels of correlation $\rho$. The upper left graph shows the price of the relax certificate, the upper right graph gives the survival probability until the time to maturity. The prices of the knock-out-component and knock-in-component are shown in the lower left and right graph, respectively. The parameters for the base case are $m=0.5, \delta=0.11, \underline{\underline{T}}=\{1,2,3\}$, $S_{0}^{(1)}=S_{0}^{(2)}=1$ and $r=0.05$. 


\section{Price Upper Price Bound}

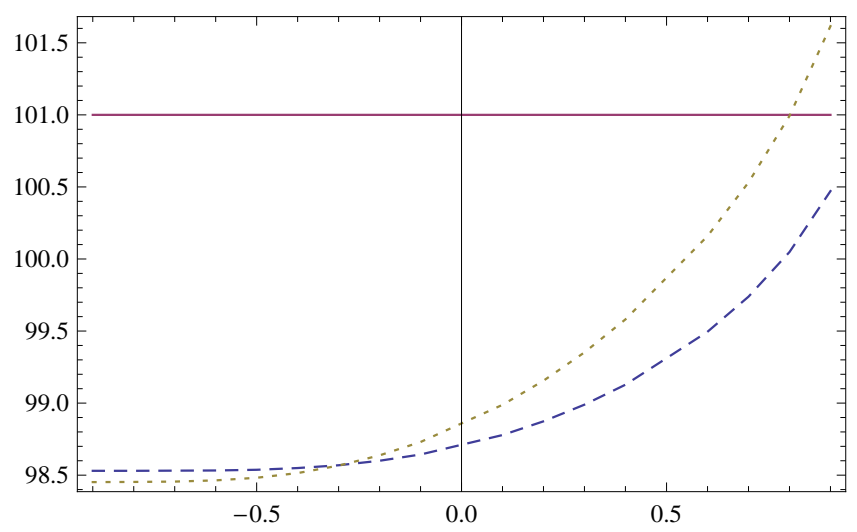

Figure 2: Upper Price Bound of Z1 and Z2

The figure shows the issue price (solid line) and the upper price bounds for Z1 (dotted line) and Z2 (dashed line) which result from Theorem 1 as a function of the correlation between the underlyings. The implied volatilities are given in Table 2. 